\title{
تقدير المحتوى الميكروبي للحوم الاجاج المحلي والمستورد في اسواق مدينة بنغازي، ليبيا
}

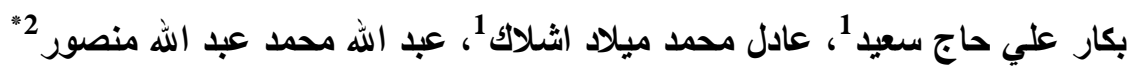

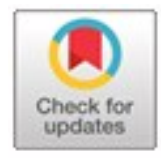

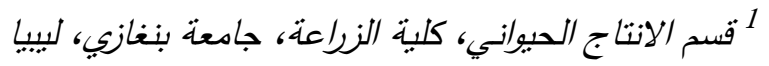

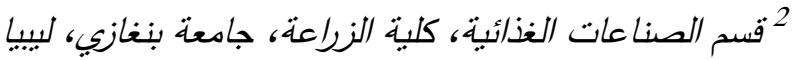

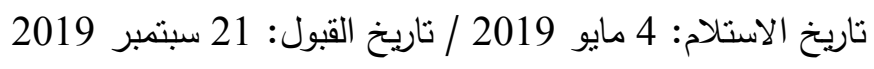
https://doi.org/10.54172/mjsc.v34i3.289:Doi

المستخلص: استهدفت الدراسة تقييم النوعية البكتيرية للحم الدجاج المسوق في 5 مناطق من مدينة بنغازي خـلال موسم الصيف من خلال تقدير أعداد بعض أدلة التلوث، والمتضمنة العدد الكلي للبكتيريا الهوائية وبكتيريا القولون وبكتيريا المكورات العنقودية الذهبية (Staphylococcus aureus) كما شملت الدراسة تشخيص وعزل بكتيريا السالمونيلا (Salmonella). أظهرت نتائج

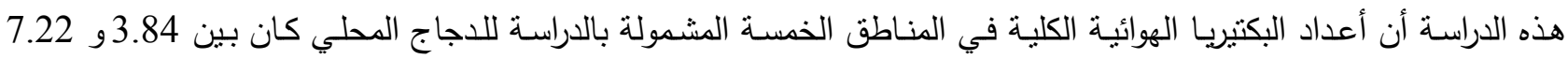
وبمتوسط 6.02 لو 10 و.ت.م/جم وفي الدجاج المستورد بين 4.49 و 6.73 ومتوسط 5.94 لو 10 و .ت .م/جم ، أما بكتيريا القولون فبلغت في المحلي بين 3.5 و 6.80 وبمتوسط 5.53 لو 10 و .ت.م/جم وفي المستورد بين 3.0و 6.80 وبمتوسط 5.5 لواتو 10.0

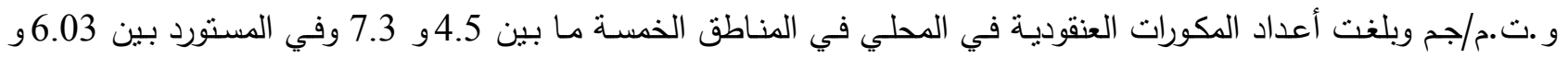
6.50 في المستورد وبمتوسط 6.40و 6.28 لو 10 و .ت .م/جم على التوالي. أجريت على العزلات اختبارات كيموحيوية للتعرف ولته

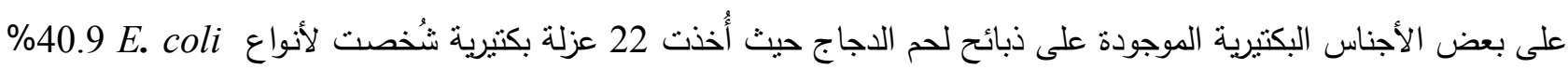

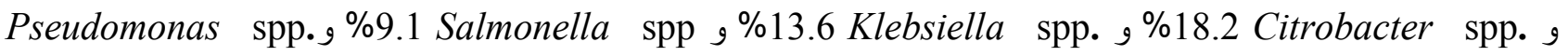

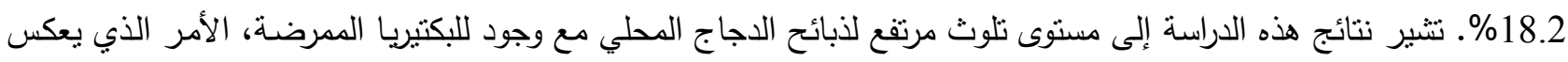

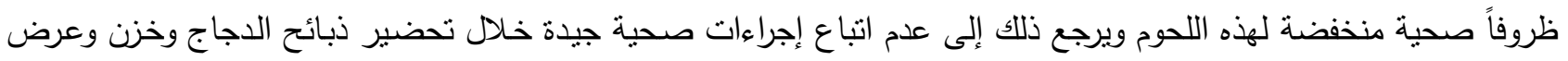
الذبائح عند البيع.

الكلمات المفتاحية: بكتريولوجية اللحوم، لحوم الدواجن، السالمونيلا، الميكروبات الممرضة، بنغازي.

وهي البكتيريا وبدرجة أقل الفطريات التي لا تعتبر ذات أهمية

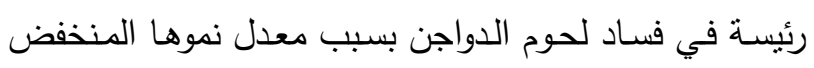
نتيجة للظروف التتافسية التي نتتج عن سيادة البكتيريا السالبة

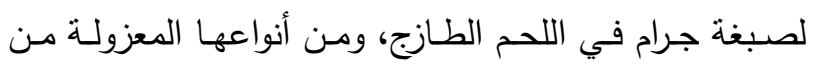

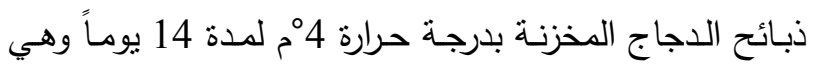
Kluyveromyces و Cryptococcus Candida

Hinton) Yarrowia,Zygosaccharomyces, وآخرون، 2002). يمكن تقسيم البكتيريـا الملوثنة للحوم الدواجن إلى مجموعتين

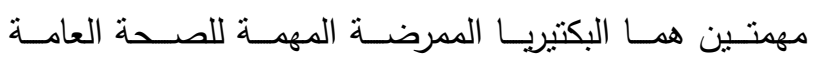

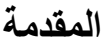

تعد لحوم الدواجن ومنتجاتها من الأغذية ذات الأهمية العالية لدى المستهلك ليس لاستساغتها ورخص ثمنها وحسب ولكن

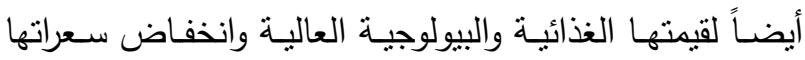

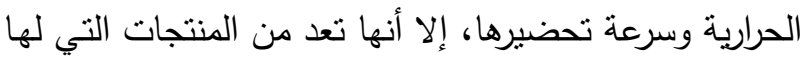

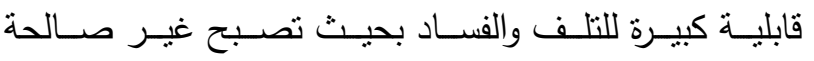

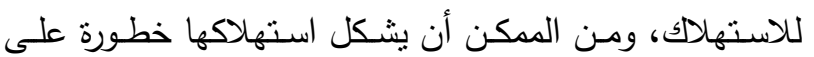
صحة المستهلك نتيجة لتسبيها بالتسمم الغذائي له.

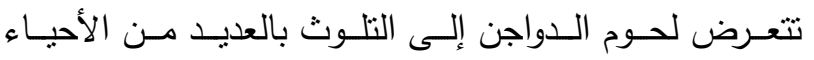

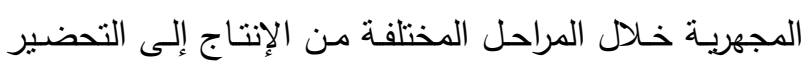


Al-Shadeedi) التلوث مثنل بـذور الحبـة السوداء أو زيتها وآخرون، 2005) وغسل أقفاص نقل الطيور بالمـاء وتركها

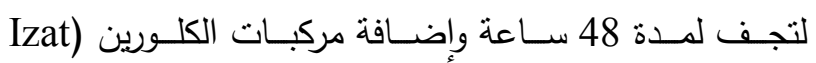

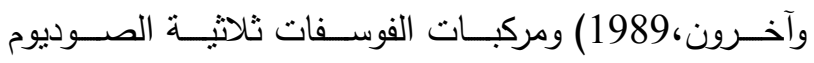

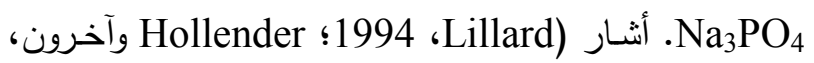
1993) إلى تأثثرات المعاملـة بهذا المركب. ومـن المركبـات التــي اســتخدمت الأوزون (Sheldon و Brown، 1986)

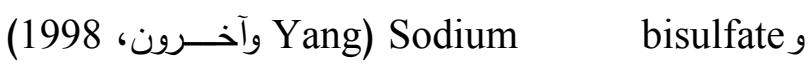
(1978 ،Ivey gobach) sorbate Potassium و و و Izat) $\mathrm{H}_{2} \mathrm{O}_{2}$

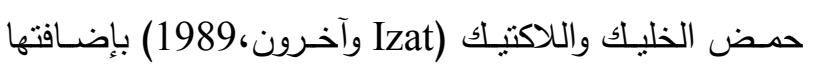

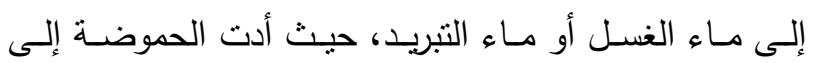

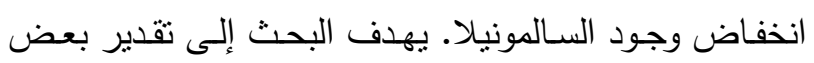
المؤشرات البكتيريـة وبعض البكتيريا الممرضـة والمسبية لفساد لحوم الدجاج المبيعة في أسواق مدينة بنغازي.

\section{المواد وطرق البحث}

العينـات: جُمعت عينات لحوم الدجاج المحلي (غير المجمد) والمستورد (المجمد) من السوق المحلي لمدينة بنغازي من 5 مناطق (A) (شارع عشرين والسلماني)، B (حي السلام)،

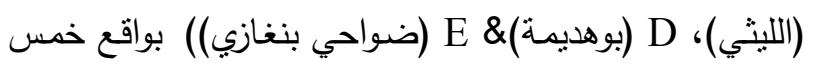
عينات من كل نوع (المحلي والمستورد) وتم جمع العينات منها

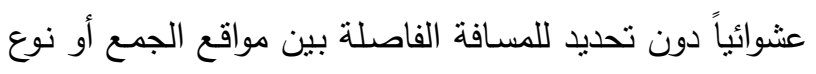

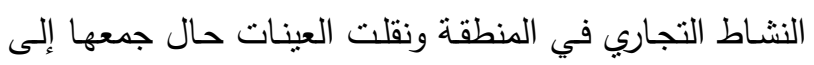
المعمل حيث نم البدء في العمل عليها وتجهيزها لعد البكتيريا

وتنتخيصها.

الفحوصات البكتيريولوجية: تقدير أعداد البكتيريا: قدرت أعداد

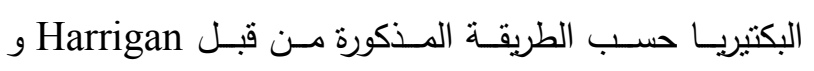
McCance لتقدير أعداد البكتيريـا الكليـة (Pour Plate Method) وبكتيريا القولون (الكوليفورم) وطريقة نشر الأطباق (Ppread (Plate Method
للمسـتهلك والمسـببة لأمـراض التسـمم الغــائي فـي الإنسـان

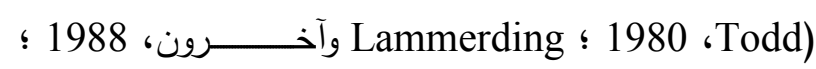
S Salmonella و S Bryan Listeria monocytogenes , Campylobacter jejuni و Escherichia coli , Clostridium perfringens 1989،Lillard) Staphylococcus aureus

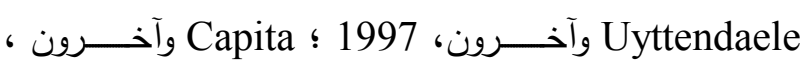

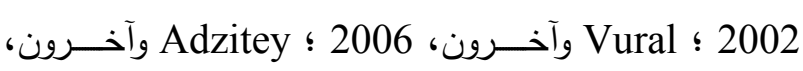

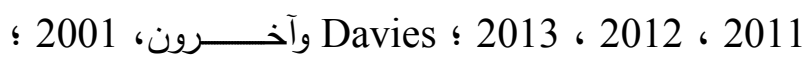
Hänel 2018) والبكتيريـاــا المسـببة للفسـاد المســؤولة عـن خســائر اقتصادية في صناعة اللحوم والمسببة لخفض العمر التخزيني

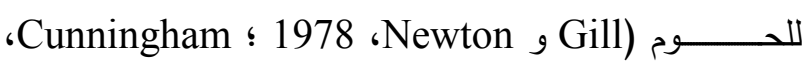
1987 ؛ Mead، 1990) ومن أكثر الأنواع شيوعاً والمرتبطة

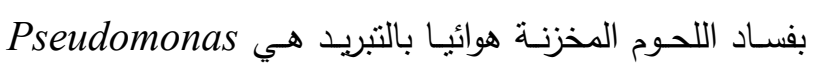
Moraxella, Enterobacter, Acinetobacter, Geornaras) Brochothrix, Flavobacterium, وآخرون، 1996). ينشـأ تلوث الذبائح من البكتيريـا المحمولة على جلد أو ريش

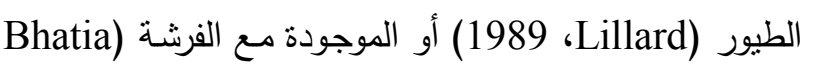

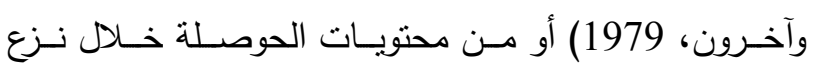

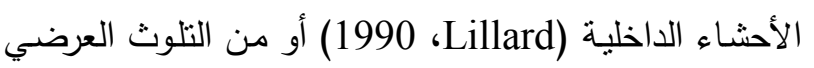
في المجازر أثناء التحضير (Rouger وآخرون، 2017). اتبعت إجراءات عدة لغرض تحسين النوعية الميكروبية للحوم

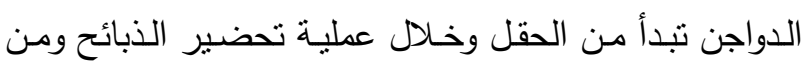

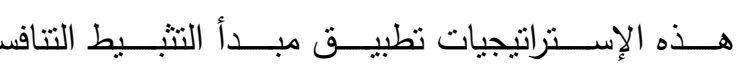
Nurmi ) أو مبدأ نورمي (Competitive exclusion) (concept Rantala العلف قبل الذبح بـ 8-12 ساعة لخفض المحتوى الميكروبي

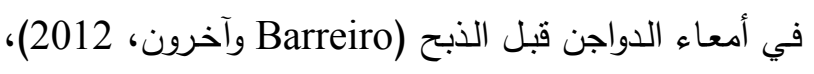
وإضـافة العديد من المواد إلى الأعلاف لهاء لها دور في خفض 


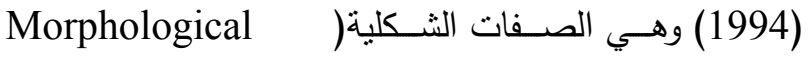
Characteristics ) والمتضــنة شكل المستعمرات وحجمها ولونها ومظهرها وحافتها والفحص المجهري ( Microscopic (Examination للتعرف على شكل وترتيب الخلايا البكتيرية

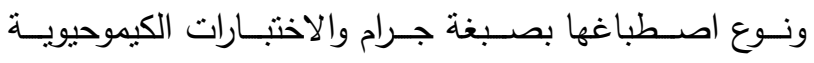
(Feng) (Biochemical Tests)

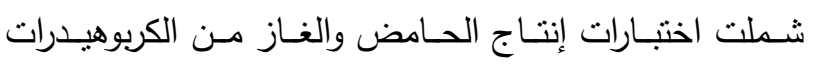

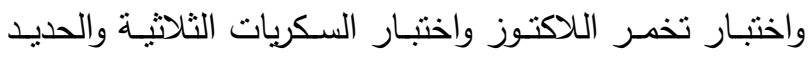

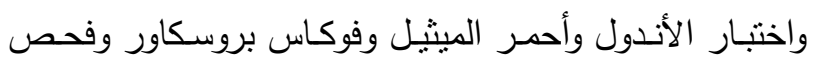
إنزيم الكاناليز والأكسيديز واستهلاك السترات.

SPSS التحيل الإحصـئي: حللت النتائج باستخدام برنامج الإحصائي النسخة 23 (Statistical package for social science version 23, IBM/SPSS) البكتبرية الدحسوبة حُوّلت لأجل التحليل الإحصائي إلى لو 10

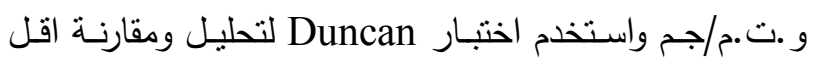
فرق معنوي L.S.D بين القطاعات وبين مصادر الدواجن

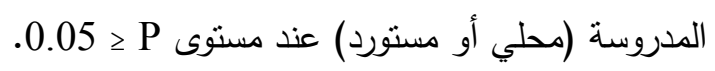

\section{النتائج والمناقثة}

يبين الجدول (1) أعداد البكتبريـا الكلية الدقدرة على سطح

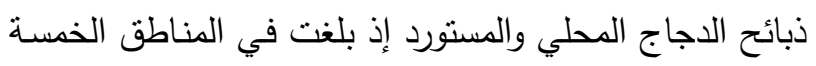

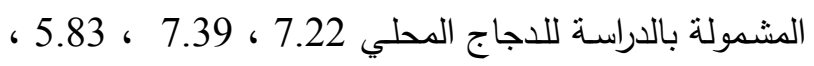

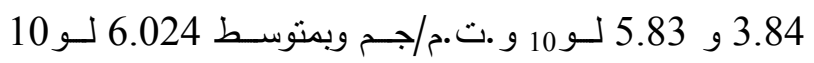

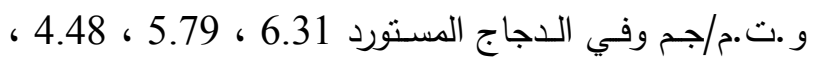

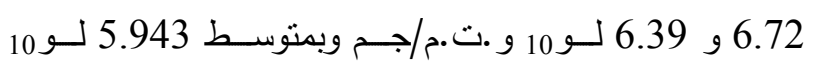

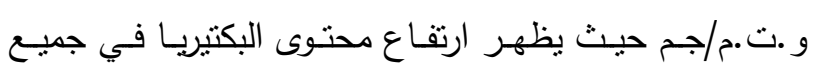

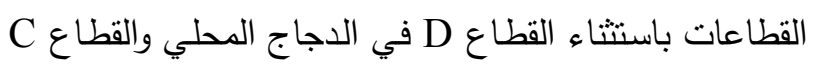

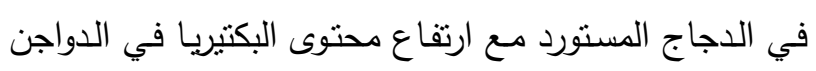
المحلية والدواجن المجمدة الدستوردة .

جدول (1). أعداد البكتيريا الهوائية الكلية (لو10 و.ت.م/جم) على ذبائح

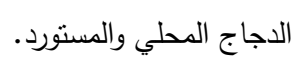

وذلك بوضـع الذيحـة في (Staphylococcus aureus) كبس نـايلون معقم يحتوي على 200 مل مـن مـاء البينون

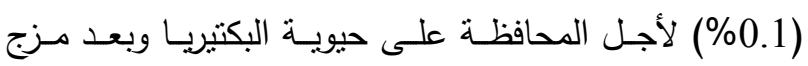

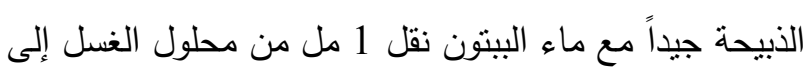

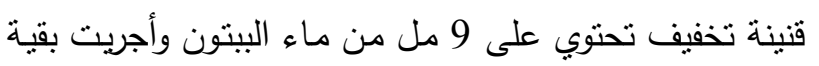
التخافيف العشرية وصولاً إلى التخفيف السادس 10 10- 10 ثن نقل 1 مل (لنقدير البكتيريا الكلية وبكتبريا القولون) و 0.1 مل الفئ

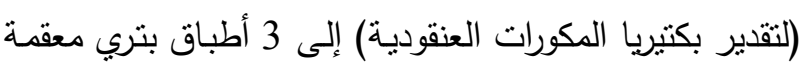
Pن من تلخفيف وصب فيها الوسط الزرعي المعقم

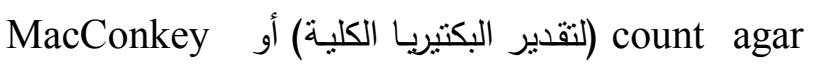
Agar حرارة 45 م تقريباً في كل طبق، أو النشر على سطح الوسط لزئ Staphylococcus medium No الزرعي المعقم 110 (لتقدير بكتيريا الككورات العنقوديـة) ثم تحريك الأطباق إلى

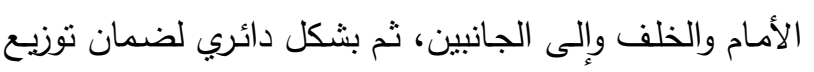

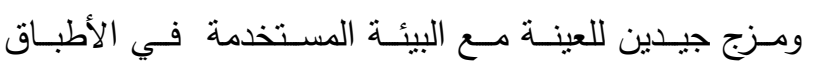

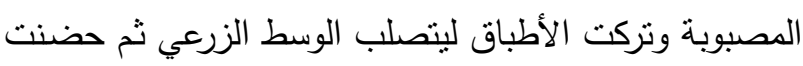

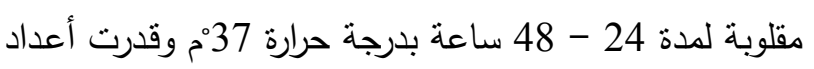

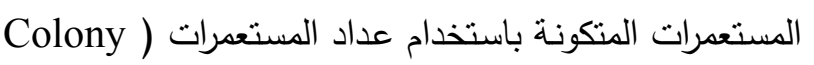
Counter في الأطباق الحاوية على 30-300 مستعمرة. عزل وتثخيص بكتيريـا السـالمونيلا: حُضِنَ محلول غسل الذبائح عند 350م لمدة 24 ساعة ثم نقل 1 مل مل منه إلى قناني

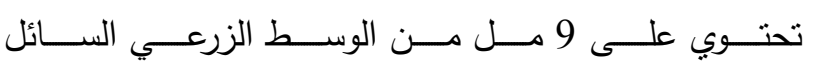

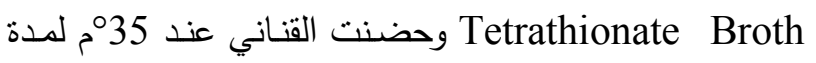

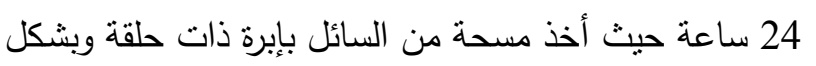

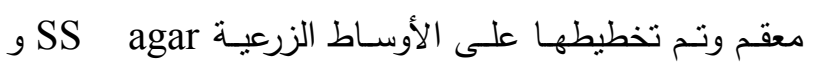
XLD agar

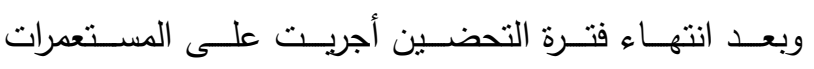

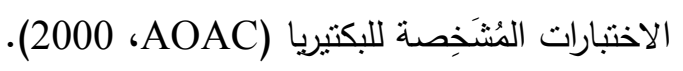

الاختبارات التشخيصية (Identification Tests): أجريت

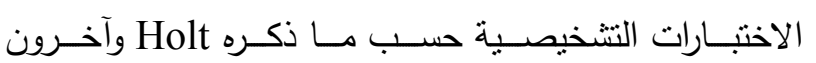


تحتوي ذبائح الدجاج المحلي والمستورد على أعداد مرتفعة من بكتيريـا المكورات العنقوديـة (الجدول 3) حيث بلغت أتح أعدادها

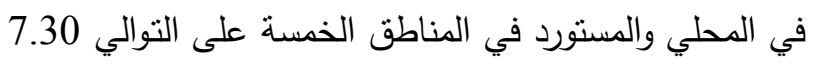

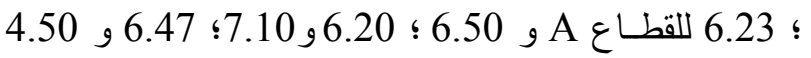

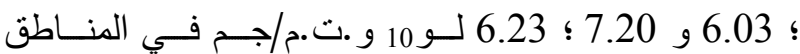
الخمسـة علـى التــوالي وبمتوســ 6.400 ؛ 6.288 لــــ 10 و وتث.م/جم جدول(3). أعداد بكتيريا المكورات العنقودية (لو10 و .ت.م/جم) على ذبائح

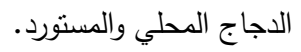

\begin{tabular}{|c|c|c|c|}
\hline متوسط القطاعات & المستورد & المحلي & القطاعات \\
\hline a $6.58 \pm 0.80$ & $\begin{array}{c}\text { a } 6.23 \pm \\
0.75\end{array}$ & $\begin{array}{c}\text { a } 7.30 \pm \\
0.00\end{array}$ & A \\
\hline a $6.35 \pm 0.16$ & $\begin{array}{c}\text { a } 6.50 \pm \\
0.00\end{array}$ & $\begin{array}{c}\text { b } 6.20 \pm \\
0.00\end{array}$ & B \\
\hline a $6.78 \pm 0.42$ & $\begin{array}{c}\text { a } 6.47 \pm \\
0.06\end{array}$ & $\begin{array}{c}\text { a } 7.10 \pm \\
0.36\end{array}$ & $\mathrm{C}$ \\
\hline b $5.27 \pm 0.85$ & $\begin{array}{c}\text { a } 6.03 \pm \\
0.06\end{array}$ & $\begin{array}{c}\text { c } 4.50 \pm \\
0.20\end{array}$ & $\mathrm{D}$ \\
\hline a $6.72 \pm 0.54$ & $\begin{array}{c}\text { a } 6.23 \pm \\
0.06\end{array}$ & $\begin{array}{c}\text { a } 7.20 \pm \\
0.10\end{array}$ & $\mathrm{E}$ \\
\hline $6.340 \pm 0.80$ & $\begin{array}{c}6.288 \pm \\
0.38\end{array}$ & $\begin{array}{c}6.400 \pm \\
1.12\end{array}$ & المتوسط \\
\hline 0.724 & 0.243 & 0.369 & L.S.D \\
\hline
\end{tabular}

المتوسطات في كل عامود المشار لها بحروف مختلفة، بينها فرق معنوي عند مستوى .0.05 $\geq \mathrm{P}$

أجريت على 22 عزلة اختبارات نتخيصية مظهرية ومجهرية وكيموحيوية للتعرف على بعض الأجناس البكتيرية الموجودة على ذبائح لحم الدجاج (جدول 4) ولئه

\begin{tabular}{|c|c|c|c|}
\hline متوسط القطاعات & المستورد & المحلي & القطاعات \\
\hline a $6.77 \pm 0.50$ & $\begin{array}{c}\mathrm{b} 6.31 \pm \\
0.00\end{array}$ & $\begin{array}{c}\mathrm{b} 7.22 \pm \\
0.07\end{array}$ & A \\
\hline a $6.59 \pm 0.88$ & $\begin{array}{c}\text { c } 5.79 \pm \\
0.01\end{array}$ & $\begin{array}{c}\text { a } 7.39 \pm \\
0.03\end{array}$ & B \\
\hline b $5.16 \pm 0.74$ & $\begin{array}{c}\mathrm{d} 4.49 \pm \\
0.09\end{array}$ & $\begin{array}{c}\text { c } 5.83 \pm \\
0.01\end{array}$ & $\mathrm{C}$ \\
\hline b $5.29 \pm 1.58$ & $\begin{array}{c}6.73 \pm \\
0.15\end{array}$ & $\begin{array}{c}\mathrm{d} 3.84 \pm \\
0.08\end{array}$ & D \\
\hline $\mathrm{ab} 6.11 \pm 0.32$ & $\begin{array}{c}\text { b } 6.39 \pm \\
0.15\end{array}$ & $\begin{array}{c}\text { c } 5.83 \pm \\
0.02\end{array}$ & $\mathrm{E}$ \\
\hline $5.983 \pm 1.08$ & $\begin{array}{c}5.943 \pm \\
0.82\end{array}$ & $\begin{array}{c}6.024 \pm \\
1.32\end{array}$ & المتوسط \\
\hline 1.087 & 0.185 & 0.094 & L.S.D \\
\hline
\end{tabular}

يوضح الجدول (2) أعداد بكتيريا القولون الموجودة على سطح

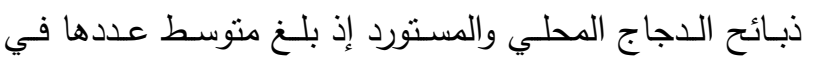

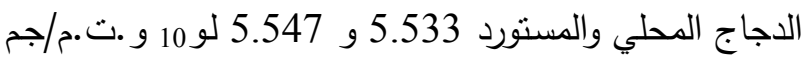
على التوالي بواقع 6.80 و 6.63 و 6.10 و 4.50 و 3.53 و 6.63.

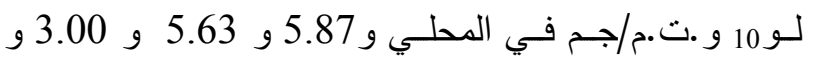

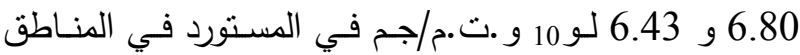

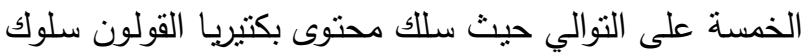
أعداد البكتيريا الهوائية نفسها من حيث عددها في القطاعات الخمسة. جدول(2). أعداد بكتبريا القولون الكلية (لو 10 و.ت.م/جم) على ذبائح الدجاج المحلي والمستورد.

\begin{tabular}{|c|c|c|c|}
\hline منوسط القطاعات & المستورد & المحلي & القطاعات \\
\hline a $6.33 \pm 0.51$ & $\begin{array}{c}\text { c } 5.87 \pm \\
0.06\end{array}$ & $\begin{array}{c}\mathrm{a} 6.80 \pm \\
0.00\end{array}$ & $\mathrm{~A}$ \\
\hline ab $6.13 \pm 0.56$ & $\begin{array}{c}\mathrm{d} 5.63 \pm \\
0.06\end{array}$ & $\begin{array}{c}\text { a } 6.63 \pm \\
0.15\end{array}$ & B \\
\hline c $3.55 \pm 0.64$ & $\begin{array}{c}\mathrm{e} 3.00 \pm \\
0.27\end{array}$ & $\begin{array}{c}\text { b } 4.10 \pm \\
0.17\end{array}$ & $\mathrm{C}$ \\
\hline b $5.15 \pm 1.82$ & $\begin{array}{c}\text { a } 6.80 \pm \\
0.00\end{array}$ & $\begin{array}{c}\text { c } 3.50 \pm \\
0.27\end{array}$ & $\mathrm{D}$ \\
\hline a $6.53 \pm 0.12$ & $\begin{array}{c}\text { b } 6.43 \pm \\
0.06\end{array}$ & $\begin{array}{c}6.63 \pm \\
0.06\end{array}$ & $\mathrm{E}$ \\
\hline $5.540 \pm 1.41$ & $\begin{array}{c}5.547 \pm \\
1.39\end{array}$ & $\begin{array}{c}5.533 \pm \\
1.49\end{array}$ & المتوسط \\
\hline 1.101 & 0.230 & 0.290 & L.S.D \\
\hline
\end{tabular}

المنتسطات في كل عمود المشار لها بحروف مختلفة، بينها فرق معنوي عند مسنوى 
جدول (4). الاختبارات الكيموحيوية لتعريف العزلات البكتبرية Holt وآخرون (1994)

\begin{tabular}{|c|c|c|c|c|c|c|c|c|c|c|c|c|c|}
\hline \multirow[b]{2}{*}{ الاختبار الكيموحيوي } & \multicolumn{4}{|c|}{ TSI } & \multicolumn{3}{|c|}{ SIM } & \multirow[b]{2}{*}{ 包 } & \multirow[b]{2}{*}{$\lesssim$} & \multirow[b]{2}{*}{3} & \multirow[b]{2}{*}{ 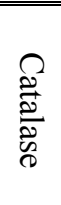 } & \multirow{2}{*}{ 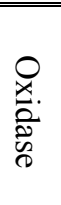 } & \multirow{2}{*}{ 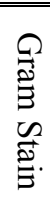 } \\
\hline & $\overline{3}$ & 司 & $\stackrel{\widetilde{I}}{\mathbb{I}^{\prime}}$ & שే & $\frac{\stackrel{D}{E}}{\stackrel{E}{\Xi}}$ & $\begin{array}{l}\frac{0}{0} \\
\frac{0}{\Xi}\end{array}$ & $\stackrel{\stackrel{D}{\Xi}}{\stackrel{D}{0}}$ & & & & & & \\
\hline E. coli & A & A & - & + & - & + & + & - & - & + & + & - & - \\
\hline Citrobacter & Alk/A & A & $\mathrm{d}$ & + & $\mathrm{d}$ & - & + & + & - & + & + & - & - \\
\hline Klebsiella & A & A & - & + & - & + & - & + & + & - & + & - & - \\
\hline Pseudomonas & Alk & Alk & - & - & - & - & + & + & - & - & + & + & - \\
\hline Salmonella & Alk & A & $\mathrm{V}$ & + & $\mathrm{V}$ & - & + & - & - & + & + & - & - \\
\hline
\end{tabular}

Alk: تفاعل قلوي، A: تفاعل حامضي، MR: أحمر الميثيل، VP: فوكاس بروسكاور، +: نتيجة موجبة، -: نتيجة سالبة، d: نتيجة مختلفةV: متغير

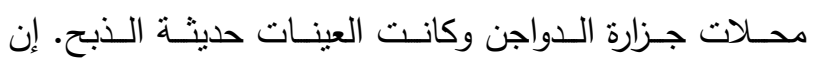

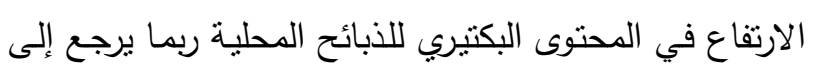

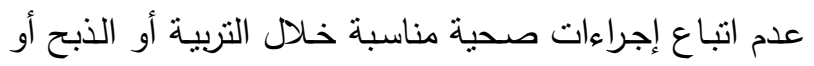

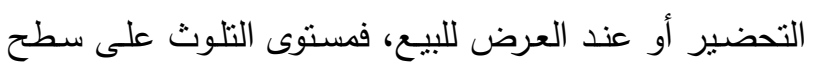

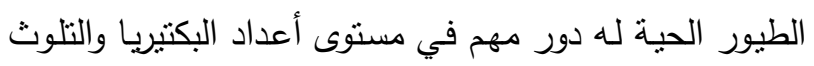

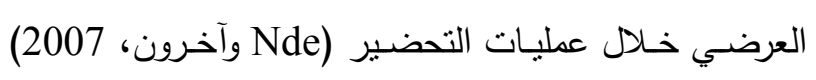
كما أن عدم سحب العلف من أمام الطيور قبل الذبح يسبب

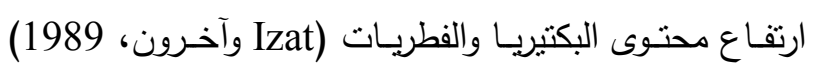

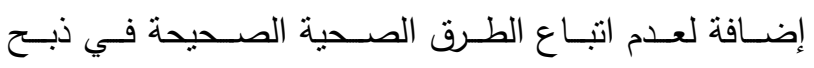

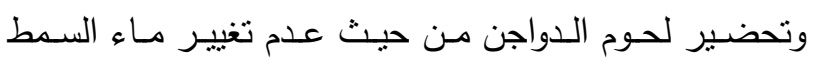

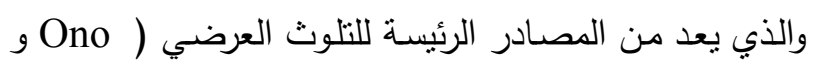
Yamamoto

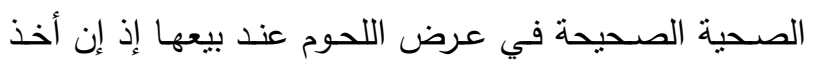
العينات كان في موسم الصيف ودرجات الحرارة مرتفعة، والتي

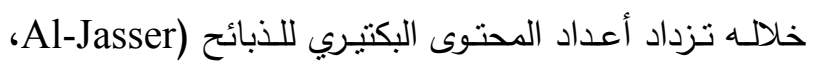

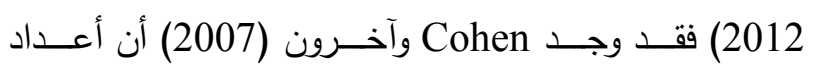

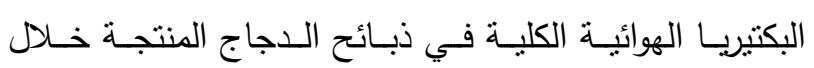
الأثهر الحارة كانت أعلى بمقدار 18\% مقارنة بذبائح الدجاج المنتجة خلال الأشهر الباردة. وجد Yashoda وآخرون (2001) أن أعداد البكتيريا الهوائية الكلية والمحبة للبرودة بعد 48 ساعة من الخزن بدرجة حرارة

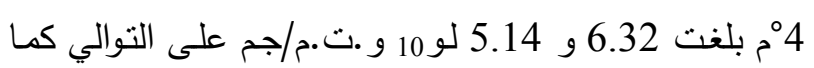

ومسن خـالل تلـك الاختبـارات كانـت البكتيريـا وتوزيعهـا في

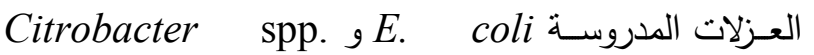
و Pseudomonas spp. و Klebsiella spp. Salmonella spp 18.2 و 9.1\% على التوالي (الجدول 5:5). جدول (5). نسب وأنواع البكتيريا المعزولة حسب الاختبارات الكيموحيوية

\begin{tabular}{|c|c|c|}
\hline$\%$ & عدد العزلات & الأجناس \\
\hline 40.9 & 9 & E. coli \\
\hline 18.2 & 4 & Citrobacter spp. \\
\hline 13.6 & 3 & Klebsiella spp. \\
\hline 18.2 & 4 & Pseudomonas spp. \\
\hline 9.1 & 2 & Salmonella spp. \\
\hline 100 & 22 & المجموع \\
\hline
\end{tabular}

\section{المناقشة}

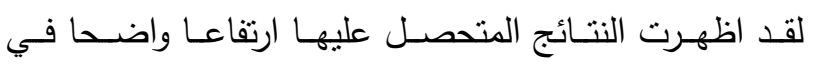
المحتوى الميكروبي الكلي لذبائح الدجاج المحلي والمسنورد

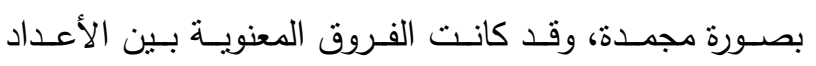

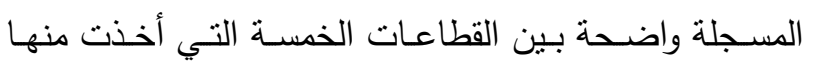
العينات حيث كان القطاع B الأعلى في محتوى البكتيريا

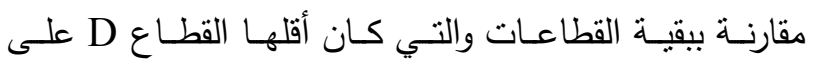

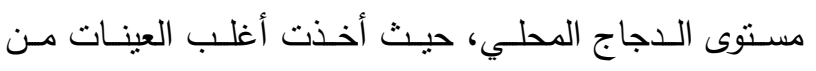


و .ت.م/جم (2 - 4 لو 10 و.ت.م/جم). ولم تشر المواصفات

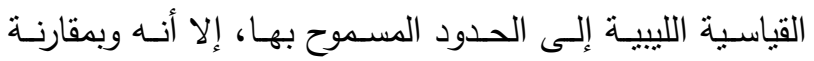

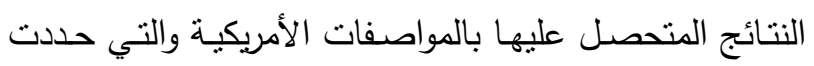

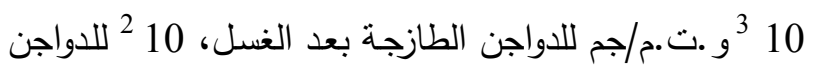
المجمدة فإن كل العزلات المدروسة فاقت هذه الحدود.

أثنارت النتائج إلى ارتفاع أعداد بكتيريا المكورات العنقودية في

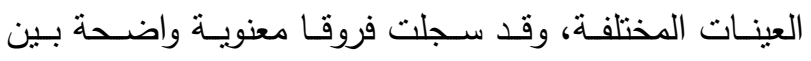
القطاعات الخمسة على مستوى عينات الدجاج المحلي، بينما لـم يكـن هنـاك فـرق معنـوي بـين عينـات الــجاج المسـتورد

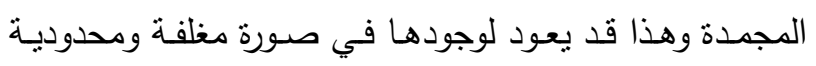
تعرضـها للوسط الخـارجي أثثاء العرض والتداول. وقد أثنـار Chaiba العنقودية في ذبائح الدجاج المبيعة في المحلات المختلفة في نظافتها ومواقعها كانت 1.85 -4.64 لو 10 و .ت. .م/جم. وجد Cohen

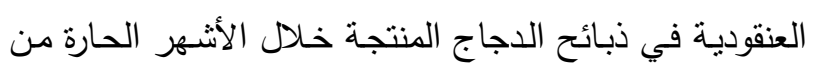

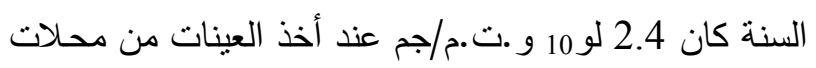

البيع.

إن البكتيريـا الأكثر أهميـة هدـا بكتيريـا Pseudomonas و Salmonella مenkitanarayanan) مسببة للنسمم الغذائي (Shu-Kee) وآخرون، 2015)، وقد يكون مصدر بكتيريا السالمونيلا من أقفاص وصناديق النقل

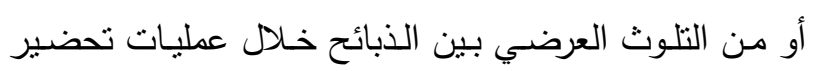

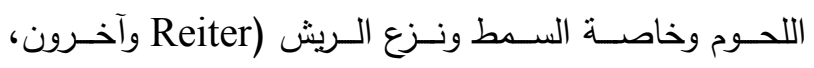

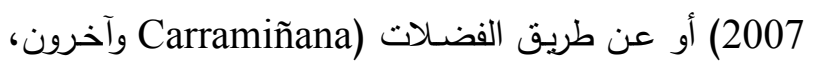
1997) وعمومـا فـإن نسـبة وجـود السـالمونيلا على ذبـ ذبـائح

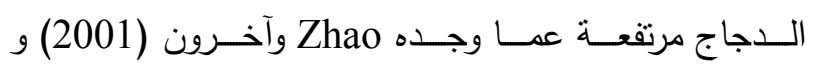

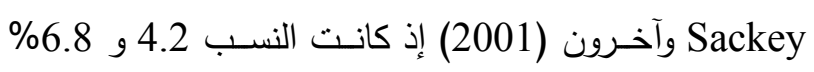

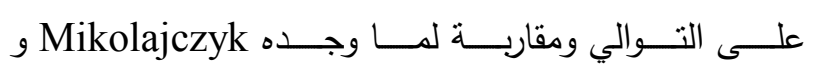

وجد Chaiba وآخـرون (2007) أن أعداد البكتيريـا الهوائيـة

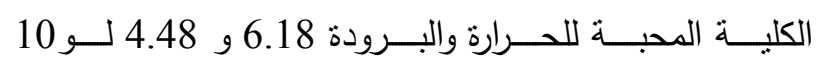

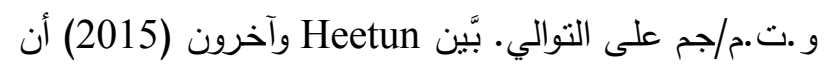
متوسط أعداد البكتيريا الكلية على ذبائح الدجاج الطازج كانت

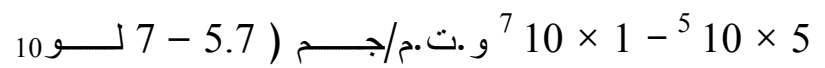
و .ت م.م/جم).

بمقارنــة نتـائج الدراســة مـع المواصـفات القياسـية الليبيــة LNCSM مـن قطاعـات B \& A لأقصسى قيمـة للحـد الميكروبـي التـي

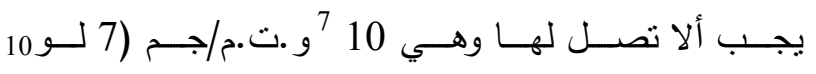
و .ت.م/جم) بينما كانت عينات باقي القطاعات ضمن الحدود المقبولة والمسموح بها وهي عدم تجاوز حد 10 كُ و.ت. 10 .م/جم

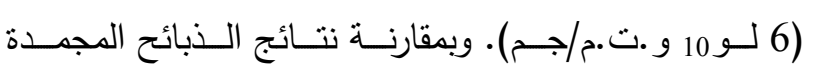

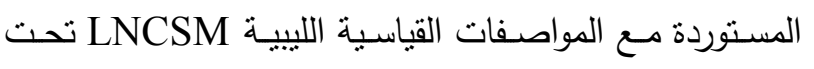

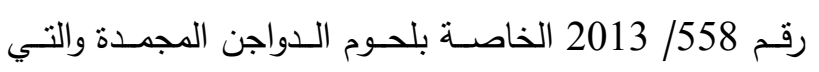
نصت على أن الحد الميكروبي المطلوب تحقيقه في المنتج هو 10 و و.ت.م/جم يلاحظ أن 60\% من الع العينات المستوردة

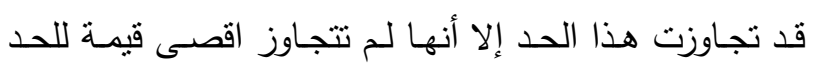

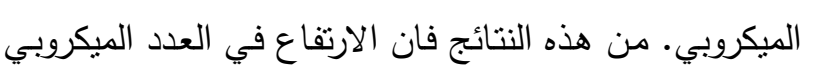

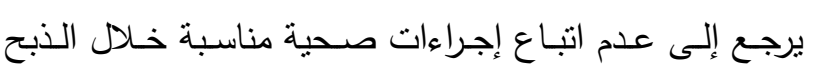
والتحضير وعند العرض للبيع.

يتضح من النتائج (جدول:2) ارتفاع محتوى الذبائح من بكتبريا

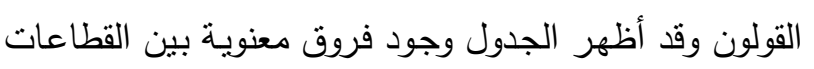

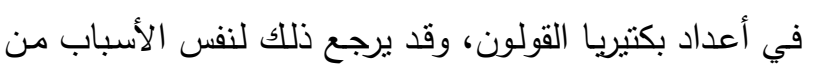
عدم اتباع الإجراءات الصحية الصحيحة في تحضير الذبائح

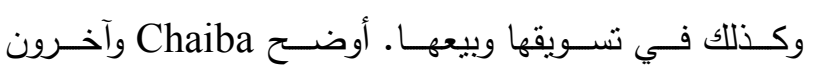
(2007) في دراستهم التي أجريت على ذبائح الدجاج المنتجة

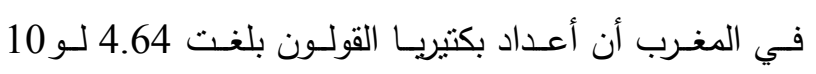

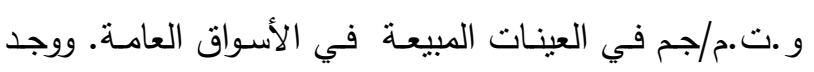

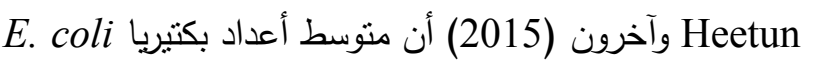

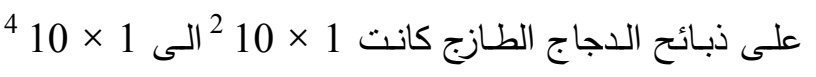




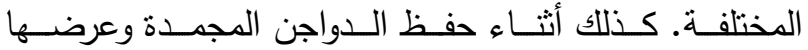
وتسويقها. وهذا يتطلب أن تكون الرقابة على كل نقاط التعامل مـع لحسوم الـدواجن مـن قبـل جهـات الاختصــاص منواصـلة ومستمرة وحازمة في تطبيق الثروط الصحية بدءاً من العاملين

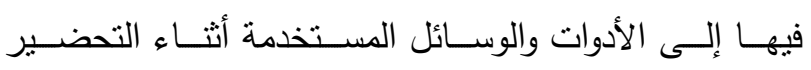
والتجهيز وكذللك أثناء التخزين (الدواجن المستوردة والمجمدة) والتسويق والعرض، مع ضرورة تثقيف العاملين في هذا المجال بالوسائل والأساليب الصحية للعمل.

\section{المراجع}

Adzitey, F. (2011). MiniReview: Effect of pre-slaughter animal handling on carcass and meat quality. Int. Food Res. J., 18: 485-491.

Adzitey, F. and Huda N. (2012). Effects of post-slaughter carcass handling on meat quality. Pak. Vet. J., 32:161-164

Adzitey, F., Liew C.Y., Aronal A. P. and Huda N. (2012). Isolation of Escherichia coli from ducks and duck related samples. Asian J. Anim. Vet. Adv., 7:351-355.

Al-Jasser, M. S.(2012). Effect of cooling and freezing temperatures on microbial and chemical properties of chicken meat during storage. Journal of Food Agriculture and Environment 10(1):113-116.

Al-Shadeedi, M. J., Al-Mashhadani, J. H., AlNidawi, N. A., Al-Shadeedi, S. M. and Al- Obaidi, F. A. (2005). Effect of adding black seed (Nigella sativa) or its oil on some microbial groups of broiler flora. The Iraqi Journal of Agriculture Sciences. 36(3):75- 80 .

AOAC. (2000). Official Methods of Analysis of AOAC International. 17th ed. AOAC Int., Gaithersburg, MD.
Radkowski بعد التبريد كانت موجبة للسالمونيلا.

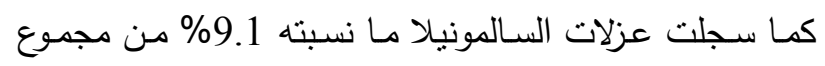
العزلات المعرفة. والتي انحصر وجودها في قطاع واحد (E) من القطاعات الخمسة وعلى مستوى الدواجن المحلية. حيث توضح هذه النسبة سوء الظروف الصحية في هذا القطاع.

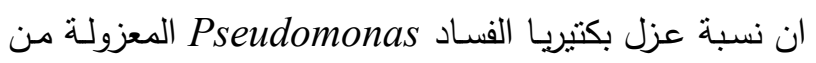

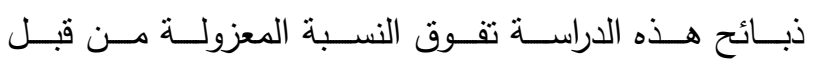
Geornaras

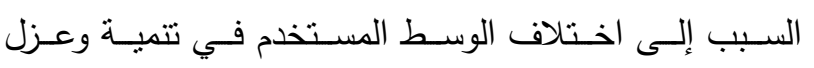
البكتيريا.

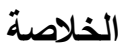

أثنتت الدراسـة أن لحوم الدواجن المحليـة المبيعـة في أسـواق

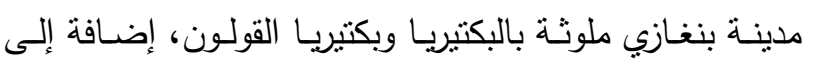
أنواع أخرى من البكتيريا. إن المصدر المحتمل لهذه البكتيريا

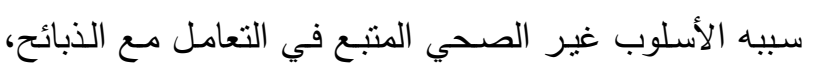

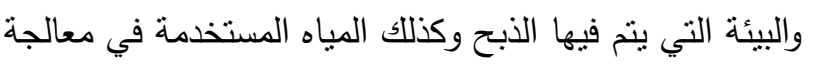

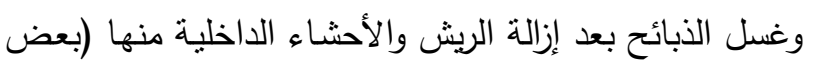

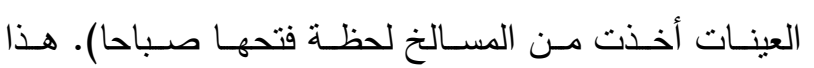

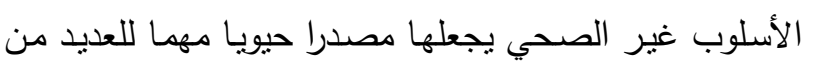
الأمراض، كما يمكنها أن تشكل مخاطر صحية خطيرة.

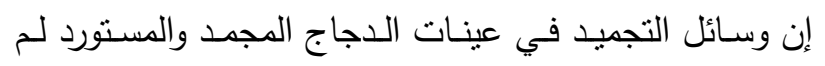
تكن كافية لأداء الغرض منها، حيث بقيت الدواجن المجمدة

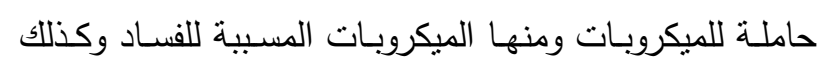

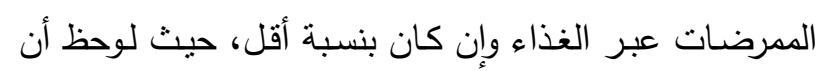

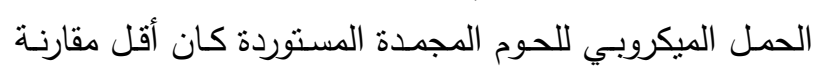
بالدواجن المذبوحة محليا.

هذه النتائج تشير إلى وجود خلل في الخطوات المنبعة للسبطرة

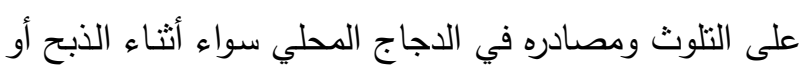
التنظيف والتغليف وحتى أثناء التتزيع والعرض في نقاط البيع 
Cohen, N., Ennaji H., Bouchrif B., Hassar M., and Karib H. (2007). Comparative study of microbiological quality of raw poultry meat at various seasons and for different slaughtering processes in Casablanca (Morocco). J. Applied Poult. Res. 16:502508.

Cox, N. A., Russell S. M., and Bailey J. S. (1998). The microbiology of stored poultry. In The Microbiology of Meat and Poultry, (Davies, A. and Board, R. eds.) pp. 266 -287, Blackie Academic \& Professional, London.

Cunningham, F. E. (1987). Types of microorganisms associated with poultry carcasses. pp. 29 - 42. In Cunningham, F. E. \& Cox, N. A. (ed.), the microbiology of poultry meat products. Academic Press, Orlando.

Cunningham, F. E. and Cox N. A. (1987). The Microbiology of Poultry Meat Products, p. ix, Academic Press, London.

Davies, R., Breslin, M., Corry, J. E. L., Hudson, W. and Allen, V. M. (2001). Observations on the distribution and control of Salmonella enterica in two integrated broiler companies. Veterinary Record 149:227-232.

Feng, P., weagant, S., and Grant M. (2002). "Enumeration of Escherichia coli and the coliform bacteria." In Bacteriological Analytical Manual (8th Ed.). FDA/Center for Food Safety \& Applied Nutrition. http://www.cfsan.fda.gov/ ebam/bam4.html.

Geornaras, I., de Jesus A. E., van Zyl E., and von Holy A. (1996). Bacterial populations associated with poultry processing in a South African abattoir. Food Microbiology 13:457-465.

Gill, C. O., and Newton K. G. (1978). The ecology of bacterial spoilage of fresh meat
Barreiro, F. R., Baraldi-Artoni S. M., Pinto, F. R., Barbosa, M. M. C., Barbosa, J. C. and Amaral, L. A. (2012). Influence of chlorine added to drinking water during the preslaughter feed withdrawal on microbiology and morphology of the broiler gastrointestinal tract. Poultry Science, 91(11):2778-2784

Berrang, M. E., Ladley S. R., and Buhr R. J. (2001). Presence and level of Campylobacter, coliforms, Escherichia coli, and total aerobic bacteria recovered from broiler parts with and without skin. J Food Prot. 64:184-188.

Bhatia, T. R. S., McNabb G. D., Wyman H. and Nayar G. P. S. (1979). Salmonella Isolation from Litter as an Indicator of Flock Infection and Carcass Contamination. Avian Diseases 23:838-847.

Bryan, F. L., and Doyle M. P. (1995). Health risks and consequences of Salmonella and Campylobacter jejuni in raw poultry. $J$ Food Prot. 58(3):326-344.

Capita, R., Alonso C.C., Rodríguez-Pérez, R., Moreno, B. and García-Fernández, M. C. (2002). Influence of Poultry Carcass Skin Sample Site on the Effectiveness of Trisodium Phosphate against Listeria monocytogenes. J Food Prot. 65(5):853856.

Carramiñana, J. J., Yangüela J., Blanco D., Rota C., Agustín A. I., Ariño A., and Herrera A. (1997). Salmonella incidence and distribution of serotypes throughout processing in a Spanish poultry slaughterhouse. J Food Prot. 60:1312-1317.

Chaiba, A., Filali F. R., Chahlaoui A., Bencheikh R. S., and Zerhouni M. (2007). Microbiological Quality of Poultry Meat on the Meknès Market (Morocco). Internet J. Food Safety. 9:67-71. 
Koch, A. G., Christensen H., Sørensen P. E., and Meinert L. (2009). Requirements to shelf-life of fresh meat and meat products. The $55^{\text {th }}$ International Congress of Meat Science and Technology (ICoMST), Copenhagen, Denmark, 16-21 August.

Lammerding, A. M., Garcia M. M., and Mann E. D., (1988). Prevalence of Salmonella and thermophilic Campylobacter in fresh pork, beef, veal and poultry in Canada. $J$. Food Prot. 51:47-52

Lillard, H. S. (1989). Incidence and recovery of salmonellae and other bacteria from commercially processed poultry carcasses at selected pre- and post-evisceration steps. J. Food Prot. 52:88-91.

Lillard, H. S. (1990). The impact of commercial processing procedures on the bacterial contamination and cross-contamination of broiler carcasses. J. Food Prot. 53:202207.

Lillard, H. S. (1994). Effect of Trisodium Phosphate on Salmonellae Attached to Chicken Skin. J. Food Prot. 57(6): 465469

LNCSM. Libyan National Center for Standardization and Metrology. (2009). Chilled chicken meat, specifications 557/2009. LNCSM.

LNCSM. Libyan National Center for Standardization and Metrology. (2013). Frozen chicken meat, specifications 558/2013. LNCSM.

McMeekin, T. A. (1982). Microbial spoilage of meats. In Developments in food microbiology ed. Davies, R. pp. 1-40. London: Applied Science Publishers.

Mead, G. C. (1990). Standardized method for determining the microbiological condition of processed poultry in relation to poten- at chill temperatures. Meat Sci. 2:207217.

Hänel, C. M., and Atanassova V. (2007). Impact of different storage factors on the survivability of Campylobacter jejuni in turkey meat. FEMS Immunol. Med. Microbiol. 49:146-148.

Harrigan, W. F. and McCance, M. E. (1976) Laboratory Methods in Food and Dairy Microbiology. Academic Press Inc. Limited, London.

Heetun, I., Goburdhun D., and Neetoo H. (2015). Comparative microbiological evaluation of raw chicken from markets and chilled outlets of Mauritius. J. World's Poult. Res. 5(1):10-18.

Hinton, A., JR., Cason J. A., and Ingram K. D. (2002). Enumeration and identification of yeasts associated with commercial poultry processing and spoilage of refrigerated broiler carcasses. $J$ Food Prot. 65(6):993-998.

Hollender, R., Bender, F. G., Jenkins, R. K. and Black, C. L. (1993). Research Note: Consumer Evaluation of Chicken Treated with a Trisodium Phosphate Application During Processing. Poultry Science 72(4):755-759

Holt, J. G., Krieg Noel, R., Sneath, P. H. A., Staley, J. T., and Williams, S. T. (1994), Bergy's manual of determinative bacteriology, [ed.] Hensyl W. R. 9th ed. Baltimore, Maryland USA: Williams \& Wilkins.

Izat, A. L., Colberg M., Driggers C. D., and Thomas R. A. (1989). Effects of sampling method and feed withdrawal period on recovery of microorganisms from poultry carcasses. J. Food Prot. 52:480-483. 
Sackey, B. A., Mensah, P., Collison, E., and Sakyi -Dawson, E. (2001). Campylobacter, Salmonella, Shigella and Escherichia coli in live and dressed poultry from metropolitan Accra. Int J Food Microbiol. 71(1):21-28.

Seuna, E. and Nurmi, E. (1979). Therapeutical Trials with Antimicrobial Agents and Cultured Cecal Microflora in Salmonella infantis Infections in Chickens. Poultry Science. 58(5):1171-1174

Sheldon, B. and Brown, A. (1986). Efficacy of ozone as a disinfectant for poultry carcasses and chill water. Journal of food science 51(2):305-309

Shu-Kee E., Priyia P., Nurul-Syakima A., Hooi-Leng S., Kok-Gan C. and LearnHan L. (2015). Salmonella: A review on pathogenesis, epidemiology and antibiotic resistance, Frontiers in Life Science, 8(3):284-293

Todd, C. D. (1980). Poultry-associated foodborne disease - its occurrence, cost, sources and prevention. J. Food Prot. 43:129-139.

Uyttendaele, M. R., Neyts K. D., Lips R. M., and Devebere J. M. (1997). Incidence of Listeria monocytogenes in poultry products obtained from Belgian and French abbatoirs. Food Microbiol. 14:339 -345.

Venkitanarayanan, K., Mazhar, I. K., Cameron, F. and Berry, B. W. (1996). Detection of Meat Spoilage Bacteria by Using the Polymerase Chain Reaction. J. Food Prot. 59(8):845-848

Viljoen, B. C., Geornaras I., Lamprecht A., and von Holy A. (1998). Yeast populations associated with processed poultry. Food Microbiol. 15:113 -117. tial shelf-life. World's Poult. Sci. J. 46:14 -18 .

Mikolajczyk, A., and Radkowski, M. (2002). Salmonella spp. on chicken carcasses in processing plants in Poland. J. Food Prot. 65:1475-1479.

Nde, C. W., McEvoy J. M., Sherwood J. S., and Logue C. M. (2007). Cross contamination of turkey carcasses by Salmonella species during defeathering. Poult. Sci. 86:162-167.

Ono, K. and Yamamoto, K. (1999). Contamination of meat with Campylobacter jejuni in Saitama, Japan. Int J Food Microbiol. 47(3):211-219.

Rantala M, and Nurmi E. (1973). Prevention of the growth of Salmonella infantis in chicks by the flora of the alimentary tract of chickens. Br Poult Sci. 14(6):627-630.

Reiter, M. G. R., Fiorese M. L., Moretto G., López M. C., and Jordano R. (2007). Prevalence of Salmonella in a Poultry Slaughterhouse. J. Food Prot. 70:17231725.

Robach, M. C. and Ivey, F. J. (1978). Antimicrobial Efficacy of a Potassium Sorbate Dip on Freshly Processed Poultry. J. Food Prot. 41(4): 284-288.

Rouger, A., Tresse, O., and Zagorec, M. (2017). Bacterial Contaminants of Poultry Meat: Sources, Species, and Dynamics. Microorganisms, 5(3), 50-65.

Ruban, W., Babu, R. N., Abraham, R. J. J., Senthilkumar, T. M. A., Kumaraswamy, P., Porteen, K., and Vemala, G. (2018). Prevalence and Antimicrobial Susceptibility of Staphylococcus aureus Isolated from Retail Chicken Meat in Chennai, India. Journal of Animal Research. 8(3):423 -427 . 
Vural, A., Erkan M. E., and Yesilmen S. (2006). Microbiological quality of retail chicken carcasses and their products in Turkey. Medycyna Wet. 62:1371-1374.

Yang, Z., Li Y., and Slavik, M. (1998). Use of Antimicrobial Spray Applied with an Inside-Outside Bird washer To Reduce Bacterial Contamination on Prechilled Chicken Carcasses. J. Food Prot. 61(7):829832.

Yashoda, K. P., Sachindra N. M., Sakhare P. Z., and Narasimharao D. (2001). Microbiological quality of broiler chicken carcasses processed hygienically in a small scale poultry processing unit. J. Food Quality 24:249 - 259.

Zhao, C., Ge, B., De Villena, J., Sudler, R., Yeh, E., Zhao, S., White, D. G., Wagner, D., and Meng, J. (2001). Prevalence of Campylobacter spp., Escherichia coli, and Salmonella serovars in retail chicken, turkey, pork, and beef from the Greater Washington, D.C., area. Appl Environ Microbiol. 67(12):5431-5436. 


\title{
Evaluation of microbial contamination of domestic and imported poultry meat in Benghazi city markets, Libya
}

\author{
Bakkar Ali Haj-Saeed ${ }^{1}$, Adel M. Milad Ishlak ${ }^{1}$, Abdalla M. Abdalla Mansour ${ }^{\text {* }}$ \\ ${ }^{I}$ Department of Animal production, Faculty of Agriculture, University of Benghazi. Libya \\ ${ }^{2}$ Department of food industry, Faculty of Agriculture, University of Benghazi. Libya
}

Received: 04 May 2019/ Accepted: 21 September 2019

Doi: https://doi.org/10.54172/mjsc.v34i3.289

\begin{abstract}
The study aimed to evaluate the bacterial quality of marketed poultry meat in five areas in Benghazi during the summer season by estimating the number of some evidence of contamination, including the total number of bacteria, coliform bacteria, Staphylococcus aureus and identification of Salmonella. The results showed that the total number of bacteria in all the studied areas on domestic chicken meat was between 3.84 and 7.22, with an average of $6.02 \log _{10} \mathrm{CFU} / \mathrm{g}$, and in imported poultry between 4.49 and 6.73 and an average of $5.94 \log _{10} \mathrm{CFU} / \mathrm{g}$. Coliform bacteria was in local chicken meat between 3.5 and 6.80 averaged $5.53 \log _{10} \mathrm{CFU} / \mathrm{g}$, and in imported poultry between 3.0 and 6.80 and averaged $5.5 \log _{10} \mathrm{CFU} / \mathrm{g}$. Staphylococcus aureus in the five sectors between 4.5 and 7.3 in domestic poultry meat, and in imported poultry meat was between 6.03 and 6.50. Biochemical tests for 22 bacterial isolates found E. coli $40.9 \%$, Citrobacter spp. 18.2\%, Klebsiella spp. 13.6\%, Salmonella spp 9.1\% and Pseudomonas spp 18.2\%. The results of this study indicate that there was a high percentage of contamination in chicken carcasses, especially local chicken carcasses, with pathogenic bacteria which reflect the low health quality of this meat due to the lack of good sanitary procedures during the preparation of chicken carcasses, storage, and display of carcasses when selling.
\end{abstract}

Keywords: Meat bacteriology, Chicken meat, Salmonella, Pathogenic bacteria, Benghazi.

*Corresponding Author: Abdalla M. Abdalla Mansourabdalla.mansour@uob.edu.ly, Department of food industry, Faculty of Agriculture, University of Benghazi. Libya. 